Document downloaded from:

http://hdl.handle.net/10251/112440

This paper must be cited as:

Castelló-Palacios, S.; Garcia-Pardo, C.; Fornés Leal, A.; Cardona Marcet, N.; Vallés Lluch, A. (2018). Wideband phantoms of different body tissues for heterogeneous models in body area networks. IEEE. 3032-3035. doi:10.1109/EMBC.2017.8037496

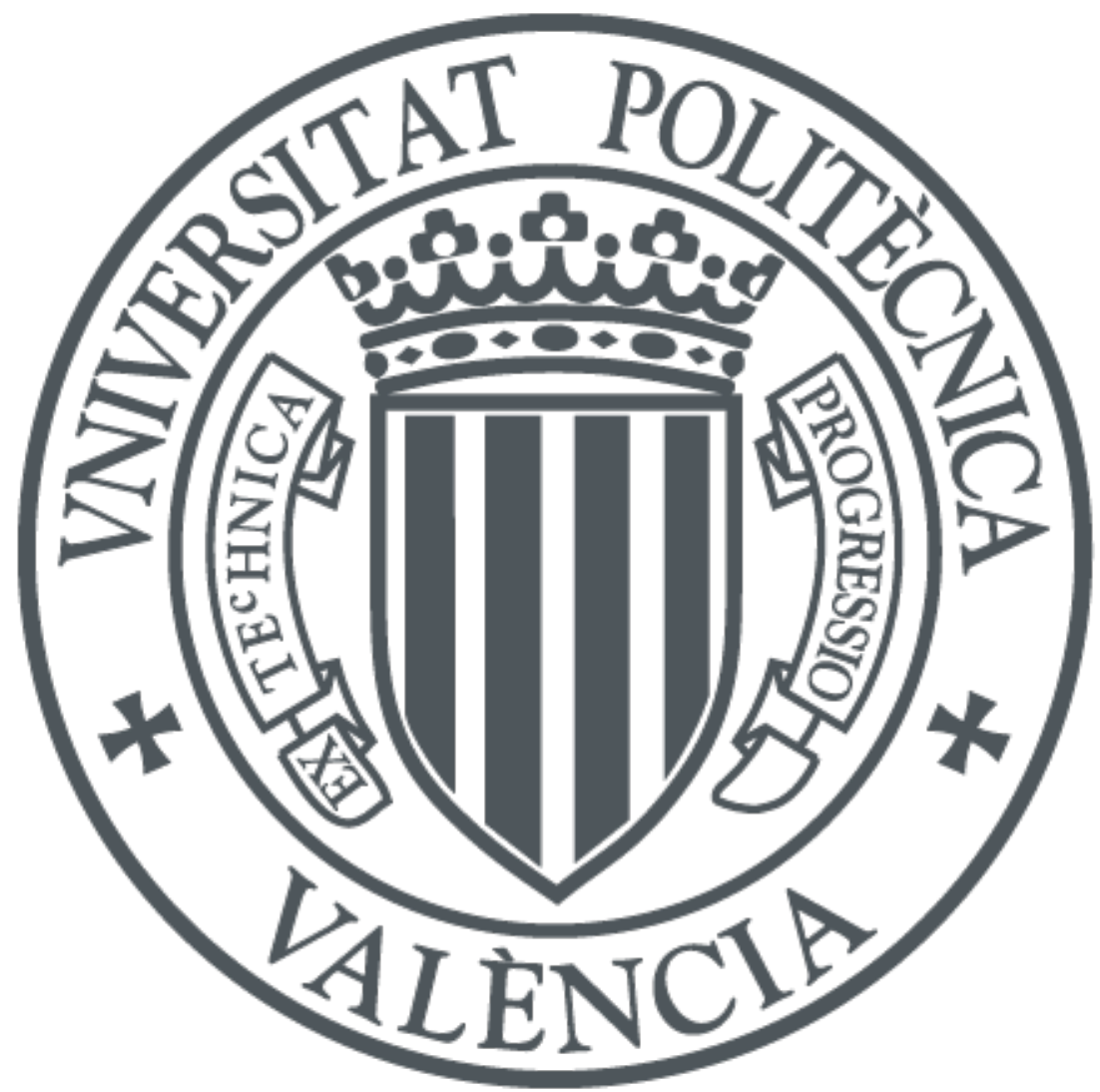

The final publication is available at

http://doi.org/10.1109/EMBC.2017.8037496

Copyright IEEE

Additional Information 


\title{
Wideband Phantoms of Different Body Tissues for Heterogeneous Models in Body Area Networks
}

\author{
Sergio Castelló-Palacios, Concepcion Garcia-Pardo, Alejandro Fornes-Leal, \\ Narcís Cardona, Member, IEEE, and Ana Vallés-Lluch
}

\begin{abstract}
One of the key issues about wireless technologies is their interaction with the human body. The so-called internet of things will comprise many devices that will transmit either around or through the human body. These devices must be tested either in their working medium, when possible, or in the most realistic one. For this purpose, tissue-like phantoms are the best alternative to carry out realistic analyses of the performance of body area networks. In addition, they are the conventional way to certify the compliance of commercial standards by these devices. However, the number of phantoms is limited in literature and they usually work in narrowband. This work aims at presenting chemical solutions that will be useful to prepare a variety of wideband tissue phantoms. Besides, the colon was mimicked under two conditions, the healthy tissue and the malignant one. This study is based on recent studies that relate the changes on its relative permittivity with cancer. They were designed on the basis of acetonitrile in aqueous solutions as it was described in a previous work. Thus, many scenarios could eventually be developed such as multilayers which imitate parts of the heterogeneous body.
\end{abstract}

\section{INTRODUCTION}

The approach of wireless connections to people's life is a fact. Current research on $5 \mathrm{G}$ communications and Internet of Things is focused on managing a large amount of networks [1] that will be present everywhere. One of the needs they will cover is health matters [2]. Body Area Networks (BAN) are expected to be used to connect sensors or embedded devices, which aim at monitoring health parameters [3]. Since the body tissues are much lossier than the air, corporal devices need to be specifically designed according to the transmitting medium. Many works are focused on characterizing the propagation channel using implantable devices (also called in-body propagation channel) and antennas that will be used to transmit this kind of data [4], [5]. These tests help to choose the best frequencies to operate or investigate the limitations of the channel. Devices that are intended to work within BANs have also to meet some specifications concerning the standards, concretely the 802.15.6-2012 - Part 15.6 [6]. All of them are encouraged to be certified for customer use with simulating media that are affected by the waves in the same way as real body would.

\footnotetext{
* Research supported by the Ministerio de Economía y Competitividad Spain (TEC2014-60258-C2-1-R) and by the European FEDER Funds.

S. Castelló-Palacios, C. Garcia-Pardo, A. Fornes-Leal, and N. Cardona are with the iTEAM, Universitat Politècnica de València, 46022 Valencia, Spain (e-mail: sercaspa@etsii.upv.es; cgpardo@iteam.upv.es; alforlea@iteam.upv.es; ncardona@iteam.upv.es).

S. Castelló-Palacios and A. Vallés-Lluch are with the Centre for Biomaterials and Tissue Engineering, Universitat Politècnica de València, 46022 Valencia, Spain (e-mail: sercaspa@etsii.upv.es; avalles@ter.upv.es).
}

Owing to the fact that multiple transmitters will also be present in the human surroundings, the effect of the body on the external signals should be taken into consideration to achieve a proper communication. Besides, the influence of this continuous radiation over the human tissues must be considered by means of, e.g., the Specific Absorption Rate (SAR). Before achieving the final product, prototypes will have to be validated in testing facilities. In all cases, interactions between human body and radio frequency waves are a key issue to be considered. Moreover, there are some other health applications that also make use of the electromagnetic waves but not for communications purposes. For instance, the microwave imaging technique, takes into account the different dielectric properties of the studied tissue for creating its image, even in those with cancer presence [7], [8].

Since human testing is not a possibility for ethical and legal issues, an artificial assembly has to be pursued. For this reason, there is an increasing interest in achieving heterogeneous models with the dielectric parameters of human body that emulate a realistic environment to test these body area networks. For these purposes, a number of materials known as phantoms have been already proposed in the literature [9]. They are used for testing the performance of antennas and systems in different scenarios [10] as well as obtaining images for cancer detection in breasts [11]. There are plenty of recipes for preparing liquids [12], gels [13] or solids [14] that simulate the electromagnetic behavior of the body at chosen frequencies [15]. However, a large part of tissues is still missing and most of these phantoms are limited for narrow bandwidths. That is why novel technologies that make use of wider frequency spectra cannot be assessed with just one phantom at the moment.

In this work, a contribution around the existing number of mimicked tissues has been pursued. Therefore, different tissues have been mimicked within a wide frequency range, from $500 \mathrm{MHz}$ up to $18 \mathrm{GHz}$. This frequency spectrum contains the main narrowband medical bands, i.e., ISM and MICS, as well as the entire promising UWB. The work was mainly focused on two issues. On the one hand, five phantoms that are not provided in literature were designed in order to provide more possibilities to create heterogeneous models. The dielectric values of human tissues were obtained from Gabriel's reference [16], which is the most commonly used. On the other hand, two more phantoms based on an elsewhere work that analyzed differences between the dielectric properties of malignant and healthy colon [7] are supplied. It may be a contribution to test future imaging techniques based on such differences. 
The different liquids were prepared considering our previous results by using acetonitrile and ethanol, since they provide similar dielectric values to those of human tissues in wide frequency ranges [17]. This is the first time that most of these phantoms are presented in literature, and the first one that all of them are usable with such accuracy in a large frequency range. This paper is organized in the following way: in section II the setup and methodology that was used characterize the liquid samples is described. In section III we present the results of the measurements of the proposed phantoms in comparison with those of their corresponding real tissues after giving their compositions. Finally, section IV summarizes the conclusions of this paper.

\section{SETUP AND Methodology}

The measurement system of the dielectric parameters consisted of a vector network analyzer (N9918A FieldFox Handheld Microwave Analyzer, frequency range: $30 \mathrm{kHz}-$ 26.5 GHz), a coaxial probe (Keysight 85070E slim form probe, working frequency: $500 \mathrm{MHz}-50 \mathrm{GHz}$ ), and a computer that managed the system and acquired the data

The system was configured in this way: the frequency range was set between $500 \mathrm{MHz}$ and $18 \mathrm{GHz}$, with 1601 recorded points, so the frequency resolution was 10.9375 MHz. The IF bandwidth and the output power were set at 3 $\mathrm{kHz}$ and $-3 \mathrm{dBm}$, respectively.

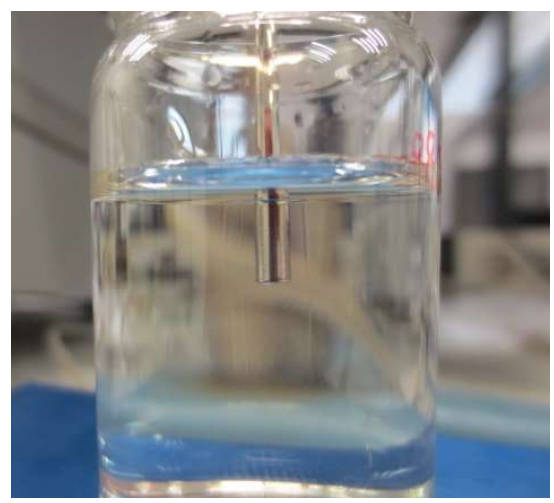

Fig. 1. Coaxial probe immersed in the liquid sample.

Using this system, we could relate the reflection coefficient from the liquid samples with their relative permittivity (1) through self-created software, following the procedure described in [18].

$$
\Gamma_{11} \rightarrow \varepsilon_{r}(\omega)=\varepsilon_{r}^{\prime}(\omega)-j \varepsilon_{r}^{\prime \prime}(\omega)
$$

For this purpose, a calibration with well-known materials had to be performed. In this case, calibration was carried out with water, methanol, air and a short termination. Next, the prepared samples were measured by immersing the coaxial probe until medium height of the vials, as depicted in figure 1. Since the temperature affects the relative permittivity, all measurements were made at 24 degrees Celsius. The accuracy of the system was determined to ensure the validity of our results. This analysis was performed by comparing the results of $0.1 \mathrm{M} \mathrm{NaCl}$ solution with those from [19], as suggested in [20]. This solution is proposed as the most appropriate one to assess the uncertainty when measuring body tissues. The results of this evaluation are separated in two frequency ranges as it is shown in table I.

TABLE I. MEASUREMENT UNCERTAINTY OF THE SYSTEM

\begin{tabular}{|l|c|c|}
\hline \multirow{2}{*}{ Frequency range } & \multicolumn{2}{|c|}{ Uncertainty } \\
\cline { 2 - 3 } & Dielectric constant & Loss factor \\
\hline $500 \mathrm{MHz}-10 \mathrm{GHz}$ & $1.24 \%$ & $2.66 \%$ \\
\hline $10 \mathrm{GHz}-18 \mathrm{GHz}$ & $1.31 \%$ & $3.34 \%$ \\
\hline
\end{tabular}

These results from the uncertainty analysis show pretty small percentages of deviation from the reference proposed in [20], so one can assume that the measurement system is highly accurate when measuring liquids whose relative permittivities are similar to those of body tissues.

\section{RESULTS}

The following solutions are based on the results of the previous work [17] in which the influence of including acetonitrile, ethanol and sodium chloride in aqueous solutions was described. First of all, regarding the phantoms that may be useful for communications purposes between implanted devices, some tissues are mimicked. These ones are intended to enlarge the possibilities of achieving heterogeneous media that will be useful to attain realistic human models. The target tissues are the wet skin, kidney, blood, cornea and grey matter. The reference values that have been pursued to match are those of Gabriel's reference [21]. Hence, after several adjustments, the resulting compositions are presented in table II. They are all weight percentages of the compounds in aqueous solutions.

TABLE II. PHANTOM COMPOSITION FOR DIFFERENT TISSUES ACCORDING TO REFERENCE [21]

\begin{tabular}{|l|l|}
\hline \multicolumn{1}{|c|}{ Tissue } & \multicolumn{1}{c|}{ Phantom composition } \\
\hline Wet Skin & $57 \%$ Acetonitrile, $13 \%$ Ethanol, $0.9 \% \mathrm{NaCl}$ \\
\hline Kidney & $50 \%$ Acetonitrile, $1 \%$ Ethanol, $1.5 \% \mathrm{NaCl}$ \\
\hline Blood & $40 \%$ Acetonitrile, $1.25 \% \mathrm{NaCl}$ \\
\hline Cornea & $49 \%$ Acetonitrile, $1 \%$ Ethanol, $1.5 \% \mathrm{NaCl}$ \\
\hline Grey matter & $50 \%$ Acetonitrile, $10 \%$ Ethanol, $1 \% \mathrm{NaCl}$ \\
\hline
\end{tabular}

Then, these phantoms are prepared, measured and compared with their analogous real tissues presented in [21]. The permittivity curves of the real tissues and the proposed phantoms of wet skin, kidney, blood, cornea and grey matter are depicted in figure 2 and figure 3, where the phantoms are shown in contrast to their analogous real tissues according to [21]. The results have been divided in two figures in order to provide a better displaying of the curves. The differences between the values are mainly due to the water content, which also differs in the phantom compositions. 

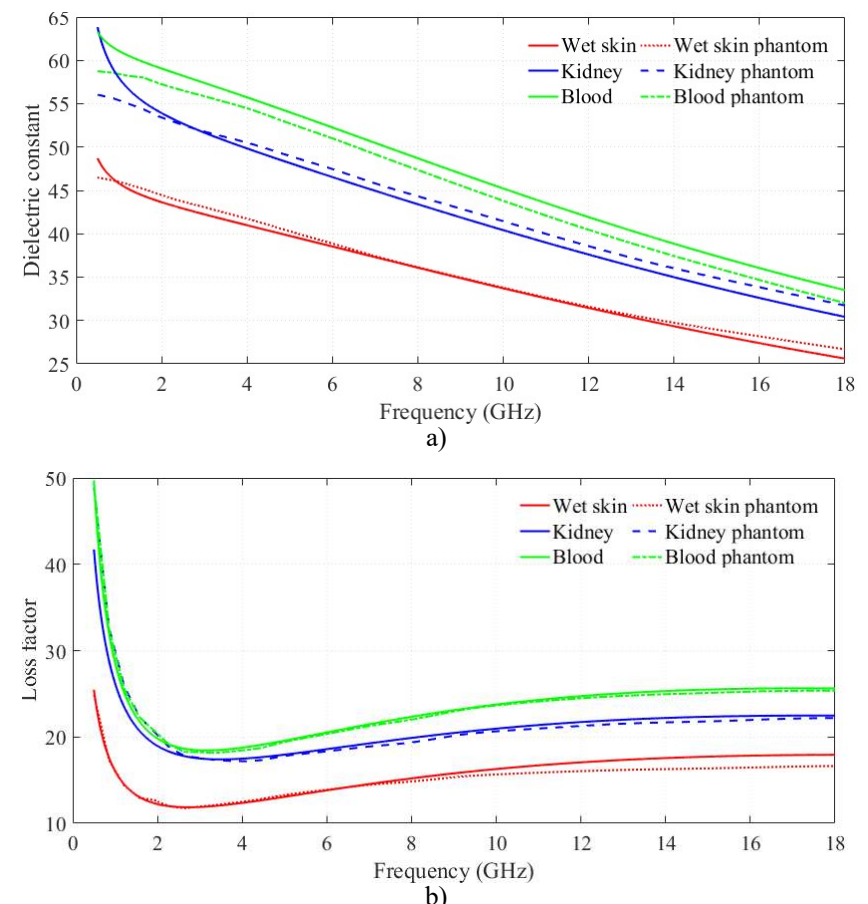

Fig. 2: Relative permittivity of the real wet skin, kidney and blood in comparison with their corresponding measured phantoms within the studied band: a) Dielectric constant b) Loss factor.

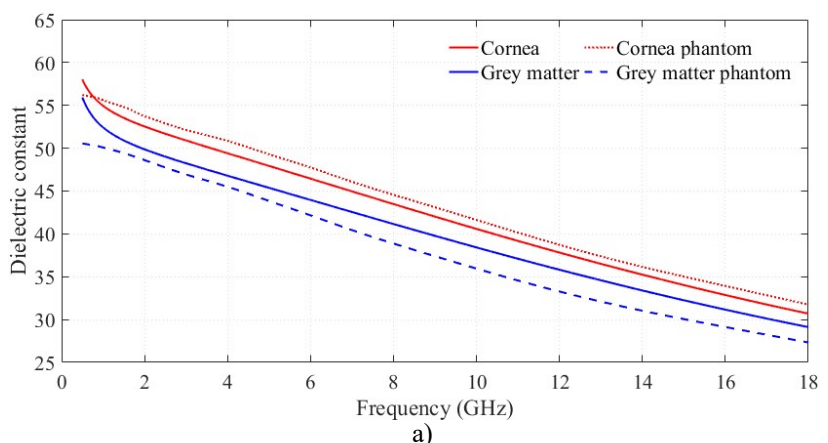

a)

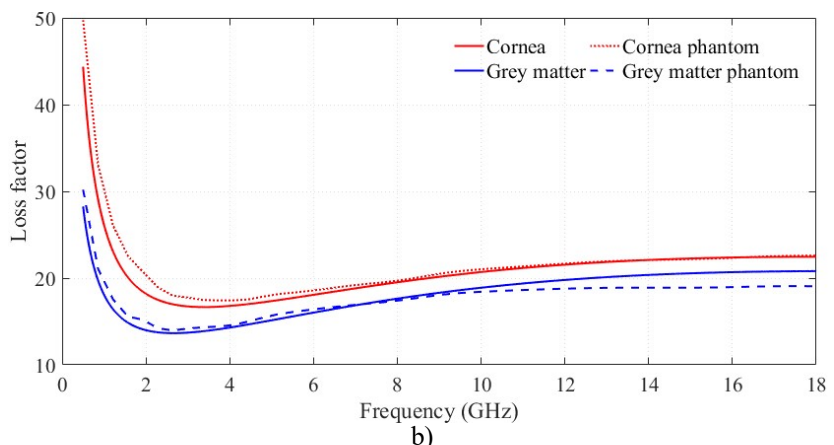

Fig. 3: Relative permittivity of the real cornea and grey matter in comparison with their corresponding measured phantoms within the studied band: a) Dielectric constant b) Loss factor.

These results along with those presented in [16] show the potential of using acetonitrile and ethanol with the aim of adjusting the location of the curves as well as their slopes. The acetonitrile reduces both parts of the relative permittivity and decreases the slope for the dielectric constant. Ethanol increases the slope of the real part whenever the slope is less pronounced due to the fact that a large amount of acetonitrile has to be included in the solution. Finally, sodium chloride increases the loss factor at frequencies below $6 \mathrm{GHz}$, at which it ceases to affect it. Thus, the development of customized tissue-like phantoms is possible.

Taking advantage of such accurate tailoring, obtaining other models, such as those provided in [7] for the colon tissue is also possible, which could be used in other medical techniques such as microwave imaging. This is made by getting the values from the experimental measurements of the Cole-Cole model with the parameters that are provided in [7]. In this way, after fitting the concentrations of the solutions, the most suitable are those presented in table III.

TABLE III. PHANTOM COMPOSITION FOR BOTH HEALTHY AND MALIGNANT COLON ACCORDING TO THE REFERENCE [7]

\begin{tabular}{|l|c|}
\hline \multicolumn{1}{|c|}{ Tissue } & \multicolumn{1}{c|}{ Phantom composition } \\
\hline Malignant colon & $42 \%$ Acetonitrile, $1 \% \mathrm{NaCl}$ \\
\hline Healthy colon & $48.5 \%$ Acetonitrile, $3 \%$ Ethanol, $1 \% \mathrm{NaCl}$ \\
\hline
\end{tabular}

The curves of relative permittivity for the measured samples are drawn in figure 4, where our proposed phantoms are faced against the model for real measurements of tissues. It can be observed that the differences between both types of tissues are maintained in the real and imaginary part.
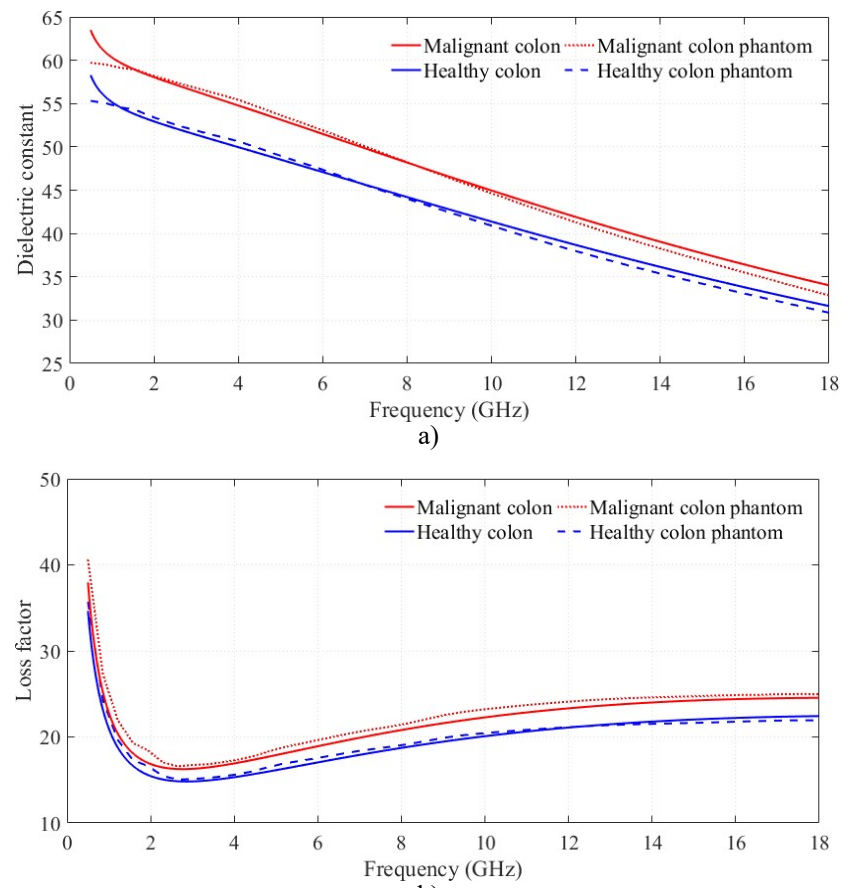

b)

Fig. 4: Relative permittivity of the real malignant and healthy colon in comparison with their corresponding measured phantoms within the studied band: a) Dielectric constant b) Loss factor. 


\section{CONCLUSION}

In this work, several novel wideband phantoms that will lead to the creation of heterogeneous models have been presented. On the one hand, phantoms of tissues from different parts of the human body that could be useful in body area networks were designed, prepared and measured within a wideband frequency range $(0.5-18 \mathrm{GHz})$. Afterwards, two additional phantoms for the healthy and malignant colon tissue were also provided. It may lead to extend the diagnosis possibilities with other techniques such as microwave imaging, which consider the dielectric properties of the targeted tissue to obtain its image. These solutions are based on previous results about combining water with acetonitrile, ethanol and sodium chloride in order to obtain wideband tissue phantoms. Tailoring the solution for the required tissue with high accuracy becomes possible thanks to this method.

The proposed phantoms contribute to the research about testing upcoming technologies which will transmit either through or around human body and will need to be validated. In fact, the studied frequency band comprises many of the ISM and MICS bands, as well as the full UWB band. It means that the main medical bands are included in this study, so future embedded sensors or capsule endoscopes can also be used in them. In addition, they are useful to establish the electromagnetic exposure or SAR in humans to certain devices in many scenarios. Furthermore, other applications like microwave imaging or MRI that must be tested for the purpose of carrying out a proper segmentation, have the solution in these phantoms. Hereinafter, more and more complex tissues could be mimicked to reproduce the full body. Besides, despite of the fact that liquids are the best option to place antennas or in-body devices inside, the jellification of these phantoms should be also considered for their use in other applications.

\section{REFERENCES}

[1] E. Hossain, M. Rasti, H. Tabassum, and A. Abdelnasser, "Evolution toward 5G multi-tier cellular wireless networks: An interference management perspective," IEEE Wirel. Commun., vol. 2, no. 3, pp. $118-127,2014$

[2] S. M. R. Islam, D. Kwak, H. Kabir, M. Hossain, and K.-S. Kwak, "The Internet of Things for Health Care: A Comprehensive Survey," Access, IEEE, vol. 3, pp. 678-708, 2015.

[3] E. Montón, J. F. Hernandez, J. M. Blasco, T. Hervé, J. Micallef, I. Grech, A. Brincat, and V. Traver, "Body area network for wireless patient monitoring," IET Commun., vol. 2, no. 2, pp. 215-222, 2008.

[4] R. Chavez-Santiago, C. Garcia-Pardo, A. Fornes-Leal, A. VallesLluch, G. Vermeeren, W. Joseph, I. Balasingham, and N. Cardona, "Experimental Path Loss Models for In-Body Communications within 2.36-2.5 GHz," IEEE J. Biomed. Heal. informatics, vol. 19, no. 3, pp. 930-937, May 2015.

[5] C. Andreu, S. Castelló-Palacios, C. Garcia-Pardo, A. Fornes-Leal, A Vallés-Lluch, and N. Cardona, "Spatial In-Body Channel Characterization Using an Accurate UWB Phantom," IEEE Trans. Microw. Theory Tech., vol. 64, no. 11, pp. 3995-4002, 2016.

[6] M. Chen, S. Gonzalez, A. Vasilakos, H. Cao, and V. C. M. Leung, "Body area networks: A survey," Mob. Networks Appl., vol. 16, no. 2, pp. 171-193, Apr. 2011

[7] A. Fornes-Leal, C. Garcia-Pardo, M. Frasson, V. Pons Beltran, and N. Cardona, "Dielectric characterization of healthy and malignant colon tissues in the $0.5-18 \mathrm{GHz}$ frequency band," Phys. Med. Biol., vol. 61 , no. 20 , pp. 7334-7346, 2016.
[8] S. Mustafa, B. Mohammed, A. Abbosh, and S. Member, "Novel Preprocessing Techniques for Accurate Microwave Imaging of Human Brain," IEEE Antennas Wirel. Propag. Lett., vol. 12, pp. 460463,2013

[9] A. T. Mobashsher, "Artificial Human Phantoms," IEEE Microw. Mag., no. July, pp. 42-62, 2015.

[10] N. Chahat, M. Zhadobov, R. Sauleau, and K. Ito, "A compact UWB antenna for on-body applications," IEEE Trans. Antennas Propag., vol. 59, no. 4, pp. 1123-1131, Apr. 2011.

[11] M. Klemm, J. A. Leendertz, D. Gibbins, I. J. Craddock, A. Preece, and R. Benjamin, "Microwave radar-based differential breast cancer imaging: Imaging in homogeneous breast phantoms and low contrast scenarios," IEEE Trans. Antennas Propag., vol. 58, no. 7, pp. 23372344, 2010.

[12] H. Yamamoto, J. Zhou, and T. Kobayashi, "Ultra wideband electromagnetic phantoms for antennas and propagation studies," IEICE Trans. Fundam. Electron. Commun. Comput. Sci., vol. E91-A, no. 11, pp. 3173-3182, Nov. 2008.

[13] A. Dabbagh, B. J. J. Abdullah, C. Ramasindarum, and N. H. Abu Kasim, "Tissue-Mimicking Gel Phantoms for Thermal Therapy Studies," Ultrason. Imaging, vol. 36, no. 4, pp. 291-316, Mar. 2014.

[14] H. Tamura, Y. Ishikawa, T. Kobayashi, and T. Nojima, "A dry phantom material composed of ceramic and graphite powder," IEEE Trans. Electromagn. Compat., vol. 39, no. 2, pp. 132-137, May 1997.

[15] C.-K. Chou, G.-W. Chen, A. W. Guy, and K. H. Luk, "Formulas for preparing phantom muscle tissue at various radiofrequencies," Bioelectromagnetics, vol. 5, no. 4, pp. 435-441, 1984.

[16] C. Gabriel, "Compilation of the Dielectric Properties of Body Tissues at RF and Microwave Frequencies," Occupational and environmental health directorate, Radiofrequency Radiation Division, Brooks Air Force Base, Texas (USA), Jun. 1996.

[17] S. Castelló-Palacios, C. Garcia-Pardo, A. Fornes-Leal, N. Cardona, and A. Vallés-Lluch, "Tailor-Made Tissue Phantoms Based on Acetonitrile Solutions for Microwave Applications up to $18 \mathrm{GHz}$," IEEE Trans. Microw. Theory Tech., vol. 64, no. 11, pp. 3987-3994, 2016.

[18] T. P. Marsland and S. Evans, "Dielectric measurements with an openended coaxial probe," IEE Proc. H Microwaves, Antennas Propag., vol. 134 , no. 4 , p. 341 , Aug. 1987.

[19] R. Buchner, G. T. Hefter, and P. M. May, "Dielectric Relaxation of Aqueous NaCl Solutions," J. Phys. Chem. A, vol. 103, no. 1, pp. 1-9, Jan. 1999.

[20] C. Gabriel and A. Peyman, "Dielectric measurement: error analysis and assessment of uncertainty," Phys. Med. Biol., vol. 51, no. 23, pp. 6033-6046, Oct. 2006.

[21] D.Andreuccetti, R.Fossi and C.Petrucci: "An Internet resource for the calculation of the dielectric properties of body tissues in the frequency range $10 \mathrm{~Hz}-100 \mathrm{GHz}$ ". Website at http://niremf.ifac.cnr.it/tissprop/. IFAC-CNR, Florence (Italy), 1997. Based on data published by C.Gabriel et al. in 1996 